\title{
Desarrollo, Causas Remotas e Historia Natural
}

\author{
Gustavo Caponi \\ Universidade Federal de Santa Catarina / CNPq
}

\begin{abstract}
Having as starting point that a proximal cause is one which effects can be registered in the states of an individual organism, in this work I will argue that what defines an ultimate cause is the fact that its effects can be registered in the evolution of lineages, and not simply in population states. This, on the other hand, not only will allow us to clarify how the developmental constraints can be understood as causes of the evolutionary phenomena; but also it will allow us to point the eminently historical character of Developmental Evolutionary Biology that Wallace Arthur lessened in Biased Embryos and Evolution. Thus, and following this latter reasoning, I will also try to show that, although the action of developmental constraints could happen beyond the limits of a population or a particular species, that does not imply that we are before a comeback of typological thought in Evolutionary Biology.
\end{abstract}

Keywords: Evolutionary developmental biology, natural history, typological thought, ultimate causes.

\section{Introducción}

Haciéndose eco de una propuesta de Gilbert, Opitz y Raff (1996), en algunos pasajes de The Changing Role of the Embryo in Evolutionary Thought, Ron Amundson (2005) señala que los actuales desarrollos de la Biología Evolucionaria Del Desarrollo podrían estar exigiéndonos una revisión, tal vez un ablandamiento o una ampliación, de la tajante distinción entre causas próximas y remotas de los fenómenos biológicos que Ernst Mayr (1998a) propuso en 1961 (cf. Beatty 1994). El estudio sobre cómo el desarrollo puede incidir en la senda y la secuencia de los cambios evolutivos no parece encajar del todo bien en la dicotomía entre Biología Funcional y Biología Evolucionaria; y esto podría estar indicando que la distinción entre esos dos órdenes causales, sobre la que se apoya la dicotomía disciplinar, tal vez no sea tan clara y estricta como Mayr pretendía.

La ontogenia es, en efecto, un fenómeno organísmico; y esto, según la categorización de Mayr (1998b, 169 y ss), coloca su estudio del lado de la Biología Funcional (cf. Amundson 2005, 212). Pero si reconocemos que los fenómenos ontogenéticos pueden permitirnos explicar parte de los fenómenos evolutivos, si reconocemos que ellos son relevantes para la Biología Evolucionaria, esto parece poner a los factores ontogenéticos en un punto intermediario entre los factores próximos y remotos

Principia 13(1): 29-50 (2009).

Published by NEL - Epistemology and Logic Research Group, Federal University of Santa Catarina (UFSC), Brazil. 
(Amundson 2005, 204), y trasformar a la Biología del Desarrollo en una disciplina que mediaría entre la Biología Funcional y la Biología Evolucionaria (Gilbert et al. 1996, 362; Amundson 2005, 225; Callebaut et al. 2007, 26). Así, y según esta posible perspectiva, la Biología del Desarrollo sería "el estudio de los cambios en la Biología Funcional", y la Biología Evolucionaria sería "el estudio de los cambios en los procesos de desarrollo" (Amundson 2005, 225).

Descreyendo que ese punto intermediario exista, y entendiendo que lo que aquí está realmente en juego no es el lugar de la Biología del Desarrollo y sí una caracterización y delimitación epistemológicamente correcta de la Biología Evolucionaria del Desarrollo, en este trabajo argumentaré que, aunque que la dicotomía de Mayr deba ser mejor formulada, tanto ella como la distinción entre Biología Funcional y Biología Evolucionaria, ameritan ser preservadas, incluso para entender en qué sentido el desarrollo puede ser relevante para el estudio de la evolución. Sostendré, en este sentido, que la dificultad no está en la propia distinción entre dos órdenes causales y entre dos órdenes disciplinares; sino en la caracterización algo imprecisa que Mayr hace del concepto de causa remota. Casi identificándolo con el de factor ecológico, Mayr nos propone una idea de ese concepto que, además de hacernos perder de vista la diferencia entre indagaciones ecológicas e indagaciones evolucionarias, nos dificulta la comprensión de cómo factores distintos a la lucha por la existencia, tales como los constreñimientos ontogenéticos, pueden llegar a tener efectos e impacto en la evolución.

Me apuro a aclarar, sin embargo, que esa posible imprecisión de Mayr era casi inevitable. Teniendo como marco de referencia la formulación neodarwiniana de la teoría evolutiva, este autor consideró a la selección natural como paradigma de toda causalidad evolutiva; y fue esto lo que lo condujo a prácticamente superponer el concepto de causa remota con el de presión selectiva. Por otra parte, y debido al hecho de que su objetivo era distinguir los campos de la Biología Evolucionaria y de la Biología Funcional en el sentido más limitado del término, Mayr descuidó el necesario deslinde entre factores y fenómenos puramente ecológicos y factores y fenómenos verdaderamente evolutivos. Hoy, en cambio, cuando los desarrollos de la Evo-Devo nos recolocan ante la relevancia evolutiva de los factores ontogenéticos, esa superposición entre lo ecológico y lo evolutivo, y esa identificación entre los conceptos de causa remota y el presión selectiva, se muestran como un obstáculo para comprender de qué modo y en qué sentido las exigencias organizacionales de la ontogenia pueden jugar el papel de factores evolutivos. Por eso, sin pretender abandonar o impugnar la distinción de Mayr, en este trabajo intentaré precisarla, y en cierto modo ampliarla, para mostrar cómo es que el estudio del desarrollo puede interesarle al biólogo evolucionario en la misma medida en que siempre le interesaron los factores relativos a la lucha por la existencia.

Así, y partiendo de la premisa de que una causa próxima es aquella cuyos efectos

Principia 13(1): 29-50 (2009). 
pueden ser registrados en los estados de un organismo individual, sostendré que lo que define a una causa remota es el hecho de que sus efectos puedan ser registrados en la evolución de linajes, y no simplemente en estados poblacionales. Esto, que no pretende ir en contra de lo dicho por Mayr sino solamente generalizarlo sin limitarlo con esa superposición entre causa remota y presión selectiva a la que aludí, no sólo nos permitirá entender en qué sentido los constreñimientos ontogenéticos deben ser comprendidos como causas remotas de los fenómenos evolutivos; sino que además nos permitirá poner en evidencia el carácter eminentemente histórico de la Biología Evolucionaria del Desarrollo que Wallace Arthur (2004a, 159) menoscabó en las últimas páginas de Biased Embryos and Evolution. Así, y siguiendo esta última línea de razonamiento, también intentaré mostrar que, aunque la acción de dichos constreñimientos ontogenéticos pueda verificarse más allá de los límites de una población o especie particular, eso no implica, como Amundson pretende $(2005,232)$, que estemos asistiendo a un retorno del pensamiento tipológico en Biología Evolucionaria.

\section{Causas Próximas y Causas Remotas}

Atendiendo, aunque sólo parcialmente, a la revisión crítica de las tesis de Mayr propuesta por André Ariew (2003), se puede presentar a la Biología Funcional y a la Biología Evolucionaria diciendo que, mientras la primera sería aquella biología ocupada en el estudio de las causas próximas que, actuando a nivel del organismo individual, nos explican cómo los fenómenos vitales se encadenan e integran en la constitución (desarrollo) y el funcionamiento (fisiología) de esas estructuras, la segunda sería esa otra biología que se ocuparía de indagar las causas remotas que, actuando a nivel de las poblaciones, nos explicarían por qué los diferentes órdenes de seres vivos presentan las características que efectivamente ahora poseen o alguna vez poseyeron (cf. Caponi 2000, 2001, 2003, 2007, 2008). Queda claro así que la Biología Funcional incluye tanto a la Fisiología cuanto a la Biología del Desarrollo (Mayr 1998b, 133; Ariew 2003, 554), y que el concepto de causa remota puede abarcar todos los factores de cambio evolutivo previstos por la versión neosintética de la Teoría de la Selección Natural: deriva genética, mutación, migración, selección sexual y la propia selección natural (cf. Ruse 1979, 45; Sober 1984, 33; Gayon 1992, 335; David \& Samadi 2000, 25; Stephens 2007, 111).

Esto último, es cierto, ya implica ir un poco más allá del concepto de causa remota conforme es presentado por Mayr; quien, como observa Ariew $(2003,557)$; parece considerarlo como un sinónimo de selección natural (cf. Mayr 1998a, 86). Pero no creo que esa ampliación nos obligue, como sugiere Ariew, a dejar de hablar de causas últimas o remotas y pasar a hablar de explicaciones evolutivas. En todo caso, podría simplemente optarse por la expresión causas evolutivas; pero no creo que se

Principia 13(1): 29-50 (2009). 
gane mucho con ese cambio terminológico: me parece mejor ser sólo un poco más cuidadosos en la delimitación de los conceptos.

Así, si entendemos por causa cualquier factor que ayude a definir y a explicar los estados y cambios de un sistema, podremos distinguir entre causas próximas que definen y explican los estados de esos sistemas físicos de alta complejidad que son los organismos individuales, y causas remotas o evolutivas que definen y explican los estados y los cambios de esos sistemas específicamente biológicos que son las poblaciones. Las causas próximas, podemos decir, son aquellas que actúan en y sobre los organismos, siendo en los tejidos, y en los estados de esos organismos en donde sus efectos pueden ser observados. Las causas remotas, mientras tanto, actúan sobre las poblaciones y es en los estados de éstas que sus efectos deben ser registrados.

Diferentemente de un fenómeno que desencadene una reacción fisiológica, o que perturbe o impulse un proceso ontogenético, los efectos de una presión selectiva, $\mathrm{ni}$ ocurren, ni pueden ser registrados, en las estructuras y estados de un organismo. Una presión selectiva actúa siempre sobre generaciones sucesivas de individuos pertenecientes a una misma población y cuyos perfiles presentan cierta variabilidad heredable; y ella siempre se traduce en una diferencia en la eficacia biológica de dos formas alternativas de un rasgo o estructura al interior de dicha población. Por eso, tanto la presión selectiva como los efectos por ella producidos, nunca podrán ser observados, ni medidos, considerando un único un organismo y su descendencia; y lo mismo ocurre con esas otras fuerzas del cambio evolutivo que son la migración, el aislamiento geográfico y la deriva genética. Hablar de una modificación en la proporción de dos alelos supone, desde el inicio, hablar de estados poblacionales que pueden ser alterados; y, en este sentido, hasta la mutación, cuando es considerada como uno de los factores capaces de desestabilizar el Equilibrio de Hardy-Weinberg, deja de ser pensada como un fenómeno organísmico, y también pasa a ser registrada como un fenómeno o una variable específicamente poblacional.

La Teoría de la Selección Natural, como lo apuntaron Elliott Sober $(1984,150)$ y Richard Lewontin $(2000,8)$, es una teoría de carácter variacional bajo cuya perspectiva los fenómenos evolutivos pueden y deben ser explicados en un nivel irreductiblemente poblacional, sin que sea posible, y tampoco necesario, considerarlos como el resultado de una acumulación de fenómenos organísmicos explicables en términos de causas próximas (ver también: Walsh 2007, 181). O como muy bien lo explicó ya hace mucho tiempo François Jacob $(1973,186-7)$ :

Lo que con Darwin transforma radicalmente la actitud hacia el mundo viviente es la manera de considerar, no ya los individuos sino amplias poblaciones. Hasta este momento, lo que se examinaban eran las variaciones a las que estaba sujeto un organismo particular para vislumbrar el tipo de transformaciones que eventualmente podría sufrir. Con Darwin, los avatares $\mathrm{y}$ desventuras que pueden afectar a un individuo o a otro, pierden interés.

Principia 13(1): 29-50 (2009). 
Es sin duda imposible reconstruir la historia de cada animal que ha vivido sobre la tierra, pero aunque se pudiese reconstruir el destino individual de cada uno de los seres del pasado, no se llegaría tampoco a deducir de ello las leyes de la evolución y la variación. El objeto de transformación no es el organismo, sino el conjunto de organismos similares que viven y se suceden en el tiempo.

Pero, si la autonomía de la perspectiva darwiniana frente al estudio de los fenómenos puramente organísmicos es la principal razón para mantener la distinción entre causas próximas y remotas, eso no debe ocultarnos el margen de ambigüedad que todavía deja la expresión poblacional. Es que, aunque sea cierto que para la Teoría de la Selección Natural los fenómenos evolutivos son siempre fenómenos poblacionales; eso no implica que todo fenómeno poblacional sea un fenómeno evolutivo, ni tampoco que cualquier variable que tenga efectos en ese dominio sea, de por sí, un factor evolutivo o una causa remota. Robert Brandon $(1990,1992)$ mostró esto en su análisis sobre los conceptos de ambiente externo, ambiente ecológico y ambiente selectivo.

\section{Precisando y ampliando el concepto de causa remota}

En el estudio biológico de poblaciones, nos dice Brandon (1992, 81), "hay por lo menos tres modos claramente diferentes de medir factores ambientales"; y cada uno de ellos supone un concepto diferente de ambiente (ver también: Brandon 1990, 47-50). Así, si estamos interesados en la población de alguna especie de hierba que crece en un campo, podríamos comenzar nuestro estudio de ese ambiente midiendo la concentración de algún elemento químico, digamos arsénico, en varias partes del terreno; pero, aunque de ese modo ciertamente obtendremos información sobre el ambiente externo de dicha hierba, puede darse el caso que la mayor o concentración de arsénico no tenga efectos sobre la distribución, la densidad y el crecimiento de la misma. Es decir: no cualquier variable ambiental es, de por sí, ecológicamente relevante; $y$, si quisiésemos salir de la duda sobre si eso ocurre o no en nuestro ejemplo, deberíamos analizar si existe o no alguna correlación entre las diferentes concentraciones de dicho elemento en distintas partes del terreno, y la distribución, la densidad y el crecimiento de la hierba en esos mismos sectores.

Así, si ese fuese el caso, si la hierba sólo nace en donde la concentración de arsénico no excede cierto nivel, y si, en dichos sectores, el crecimiento y la densidad de la misma decrecen conforme el nivel de arsénico aumenta; entonces, podremos estar seguros de haber encontrado una variable significativa del ambiente ecológico de nuestra planta. Pero, lo que aquí interesa señalar es que esto no significa que hayamos individualizado una presión selectiva actuante sobre nuestra población; es decir:

Principia 13(1): 29-50 (2009). 
eso no significa que hayamos individualizado una variable del ambiente selectivo de nuestra hierba (cf. Brandon 1990, 49, y 1992, 83). Para que ello sea así, deberíamos mostrar que en la población que estamos estudiando existe cierta variabilidad genotípica que se traduce en diferentes grados de tolerancia al arsénico (cf. Brandon 1990, 172). Esto haría que las variantes más resistentes tiendan a tener mayor éxito reproductivo que las menos resistentes; y esa circunstancia nos llevaría a considerar al arsénico como una variable evolutiva, una causa remota, que estaría controlando la composición genética de la población, sea modificándola, sea preservando una determinada proporción entre las variantes en competición.

Si no fuese así, si todas las variantes de hierba fuesen igualmente sensibles al arsénico, aunque no a otras variables ecológicas, no podríamos decir que su presencia constituya una presión selectiva para la población en estudio; y, consecuentemente, aun habiendo establecido que la presencia de arsénico incide en la distribución y en la densidad de la población, no por eso habríamos individualizado una causa remota relevante para explicar los fenómenos evolutivos ocurridos en dicha población. Queda claro, entonces, que aunque la distribución, la densidad y el crecimiento desigual de los individuos de una población son fenómenos poblacionales ecológicamente explicables por factores ambiéntales como los diferentes grados de concentración de una determinada sustancia en el ambiente; eso, por sí sólo, no transforma a dichos fenómenos poblacionales en fenómenos evolutivos, ni transforma a esas variables ecológicas en factores evolutivos. Para que esto sea así es necesario que esos factores puedan producir diferencias de éxito reproductivo entre las variantes presentes en dicha población: sólo ahí los factores ecológicos devendrán genuinas causas remotas (cf. Brewer 1994, 30).

Es decir: en sentido estricto, el fenómeno evolutivo sólo se verifica cuando, dejando de considerar a una población como un simple conjunto de organismos que explota y padece cierto ambiente que condiciona su crecimiento, su distribución y su densidad, pasamos a pensarla como un linaje cuya composición se va alterando, o preservando, a lo largo de una secuencia de generaciones (cf. Hull 1994, 197; Queiroz 1999, 50). Así, aunque no haya nada de definitivamente errado en decir que para la Teoría de la Selección Natural son las poblaciones, y no los organismos, que evolucionan; esa misma idea puede ser mejor expresada diciendo que los que evolucionan son los linajes (cf. Hull 1980, 327). Por eso, más preciso aun que definir una causa remota como aquella cuyo efecto sólo se refleja en el plano poblacional, deberíamos definirla como aquella cuyo efecto sólo se refleja en el devenir de dichos linajes.

Esta precisión adicional, sin embargo, lejos de restringir el concepto de causa remota, no hace más que ampliarlo. Es que, del mismo modo en que una población y una especie pueden ser consideradas como linajes sujetos a evolución, también pueden ser así consideradas unidades taxonómicas de orden superior. 'León' es el

Principia 13(1): 29-50 (2009). 
nombre propio de un cierto linaje; pero también lo es 'mamífero': hasta donde sabemos, esa palabra designa un grupo monofilético, un linaje particular, cuyas diferentes ramificaciones comparten un ancestro común; y, por eso, cualquier factor que pueda condicionar o afectar de algún modo la evolución de ese linaje, como un todo, debería ser considerado una causa remota. Pero, esas precisiones amplificadoras del concepto de causa remota serían incompletas si no hacemos algunas otras precisiones sobre la relación que existe entre factores ecológicos y factores evolutivos. Se trata de algunos matices que Mayr no tuvo en cuenta a la hora de explicar su concepto de causa remota y cuya omisión puede contribuir a una cierta superposición entre un análisis de la función ecológica, o rol biológico de un rasgo, y lo que cabría caracterizar como una verdadera explicación evolutiva del mismo (cf. Bock \& Wahlert $1998,131)$.

\section{Historia de una Curruca}

Mayr (1998a, 85-6), recordémoslo, distingue cuatro respuestas diferentes, pero complementarias y no mutuamente excluyentes, que pueden darse a la pregunta "ipor qué la curruca [Sylvia atricepilla] que estaba en mi casa de verano en New Hampshire, comenzó su migración hacía el sur en la noche del 25 de agosto?": una aludirá a una causa ecológica, otra a una causa genética; y las dos restantes se referirán a causas fisiológicas, intrínsecas en un caso y extrínsecas en el otro. Así, mientras estas dos últimas serían ejemplos de causas próximas, las dos primeras serían ejemplos de causas remotas; y es en su presentación de la llamada causa ecológica del comportamiento en cuestión en donde, según me parece, Mayr propicia, o pone en evidencia, una identificación incorrecta entre factores ecológicos y factores evolutivos.

Como causas fisiológicas, próximas, del comportamiento migratorio de aquella curruca, Mayr apunta a un factor interno, vinculado a la disminución cantidad de horas diarias de luz solar que predispondría al pájaro para iniciar su vuelo migratorio, y un factor externo, tal como el pasaje de una masa de aire frío y la caída de la temperatura a ella asociada, que operaría como gatillo o estímulo desencadenante de dicha conducta. La causa genética, mientras tanto, tendría que ver con la historia evolutiva de la especie: a lo largo de la misma, ésta habría adquirido la constitución neurofisiológica hereditaria responsable de dicha reacción comportamental (Mayr 1998a, 86). Pero, antes de esa segunda explicación por causas remotas, Mayr (1998a, 85-6) apunta otra, supuestamente del mismo carácter, y a la que él llama de explicación ecológica: "la curruca, que es un ave insectívora, debe migrar porque si pasa el invierno en New Hampshire morirá de hambre"; y es ahí en donde, según me parece, reside su error.

Nadie duda, por supuesto, que la disminución de la cantidad de insectos que

Principia 13(1): 29-50 (2009). 
puede ocurrir en New Hampshire durante el invierno, debe haber operado, y tal vez continúe operando, como una presión selectiva responsable de la conformación y la preservación de ese rasgo comportamental. Pero eso es lo ya supuesto en la llamada explicación genética: a lo largo de la historia evolutiva de la especie Sylvia atricepilla esa presión selectiva habría labrado y estabilizado su constitución neurofisiológica hereditaria, haciendo que, dados ciertos estímulos ambientales vinculados al cambio de estación, los especimenes de dicha ave inicien su vuelo migratorio en dirección al sur. Sin embargo, al apuntar ese factor ecológico, de por sí sólo y antes de considerar su impacto en la composición hereditaria de la especie, como siendo una causa remota, Mayr parece estar sugiriendo que todo factor ambiental que afecte el ciclo vital de un organismo constituye una presión selectiva; y eso, como lo vimos en el caso de aquellas plantas que crecían en un campo con arsénico, constituye un error.

Claro, en el ejemplo de la curruca el pasaje del factor ecológico al factor evolutivo parece tan inmediato y obvio, que resulta casi inevitable no hacer esa identificación. Pero, si lo que está en juego no es un análisis empírico sobre la relación entre la ecología y la historia evolutiva de la Sylvia atricepilla, y sí una elucidación epistemológica del concepto de causa remota, debemos intentar evitarla: debemos entender que una cosa es averiguar cuál es la ventaja ecológica que un comportamiento, o la posesión de un rasgo anatómico, puede representar para un animal; y otra cosa es hacer inferencias sobre la historia evolutiva de esas particularidades. En el primer caso, podríamos decir, estamos identificando el papel o la función que un rasgo comportamental o anatómico puede tener en el ciclo vital de un organismo; y en el segundo es muy posible, pero no necesario, que lleguemos a la conclusión de que dicho rasgo constituye una adaptación para dicha función: ambas cuestiones suelen estar muy próximas pero es importante no confundirlas.

En este sentido, puede ser muy útil comparar la caracterización de las cuatro preguntas sobre el comportamiento del curruca propuesta por Mayr, con la caracterización de las cuatro preguntas etológicas propuesta por Niko Tinbergen (1985 [1968], 168). Esta disciplina, decía Tinbergen, "ha desarrollado una serie de conceptos y términos" que, ante cualquier comportamiento de un animal, nos conducen a plantear las siguientes cuestiones:

1. ¿De qué modo este fenómeno (el comportamiento) influye en la pervivencia, en el éxito del animal?

2. ¿Qué es lo que hace que el comportamiento ocurra en un momento dado? ¿Cómo trabaja su maquinaria?

3. ¿Cómo se desarrolla la maquinaria del comportamiento cuando el individuo crece?

4. ¿Cómo han evolucionado los sistemas de comportamiento de cada especie hasta llegar a lo que son ahora? (Tinbergen 1985, 168)

La cuarta de estas preguntas, diría con toda razón Mayr, es una pregunta por

Principia 13(1): 29-50 (2009). 
las causas remotas del comportamiento. La segunda y la tercera, mientras tanto, son preguntas por causas próximas: la primera de ellas abarca las preguntas por las causas fisiológicas intrínsecas y extrínsecas del comportamiento de la curruca que fueron apuntadas por Mayr; y la otra alude a su ontogenia. Este último asunto se le pasó por alto a Mayr; pero, conforme vimos, no cabe duda que su tratamiento remite al orden de las causas próximas. El problema está en la primera de las cuatro preguntas: si razonásemos a la manera de Mayr tendríamos que decir que se trata de una pregunta por causas remotas; y, por lo tanto, de una pregunta por la evolución de ese comportamiento. Pero esto, además de llevarnos a considerar que la clasificación de Tinbergen es redundante y desordenada, también implicaría un malentendido con respecto a lo que él estaba queriendo decir cuando aludía a la influencia que un comportamiento puede tener en la pervivencia, o en el éxito, de un animal.

Conforme puede notarse en un trabajo anterior a la conferencia en donde estableció esa distinción entre las cuatro preguntas, Tinbergen suponía una distinción clara entre el análisis de cómo un comportamiento puede contribuir al éxito de un animal individual, y, el estudio de las presiones selectivas, presentes y pasadas, involucradas en la historia evolutiva de su especie (cf. Tinbergen 1979, 100-1). Es que, como ocurre en el caso de la curruca, una cosa es averiguar cuál es la ventaja ecológica que un comportamiento, o la posesión de un rasgo anatómico, puede presentar para un animal; y otra es hacer inferencias sobre la historia evolutiva de esas particularidades. Para lo primero, basta con analizar las condiciones en que el animal desarrolla su existencia, mostrando cómo el mismo contribuye a su pervivencia y desarrollo individual, a su reproducción, a la pervivencia de su prole, y eventualmente a la pervivencia de su grupo o manada; y otra cosa totalmente distinta es determinar la historia de las presiones selectivas que llevaron a la conformación de ese rasgo (cf. Tinbergen 1979, 118). Piénsese, en este sentido, lo difícil que resultó dar con una explicación darwinianamente correcta de los llamados comportamientos altruistas. La utilidad y el valor ecológico de los mismos podía ser algo obvio; pero de ahí a su explicación evolutiva había un largo camino a recorrer (ver: Williams 1966, 193 y ss; Cronin 1991, 253 y ss).

Es digno de observarse, por otra parte, que el reconocimiento de esa distancia entre el análisis ecológico del rol biológico de un rasgo y el trazado de su historia evolutiva está también implícito en la noción de exaptación propuesta por Gould y Vrba en 1982. La idea de que pueden existir estructuras heredadas que resultan útiles en el cumplimiento de cierto rol biológico, y que hasta son capaces de incrementar la aptitud de sus portadores, pero que no han sido labradas por la selección natural en virtud de dicho rol o función; nos indica que, entre el trabajo de un naturalista de campo que consigue identificar esa utilidad o conveniencia (cf. Gould \& Vrba 1998, 522), y las inferencias evolutivas que puedan hacerse sobre la filogenia de esa estructura, existe un hiato que no puede ser obviado. El clítoris hipertrofiado

Principia 13(1): 29-50 (2009). 
de la hiena moteada puede tener una utilidad en los rituales de apareamiento de esa especie; pero su evolución puede ser un efecto secundario de presiones selectivas de otra índole que "premiaron" hembras con mayor secreción de andrógenos en virtud del mayor tamaño que ellas podían alcanzar (Gould \& Vrba 1998, 529).

\section{De lo ecológico a lo evolutivo}

Puede decirse, entonces, que así como la identificación de un aspecto del ambiente que resulta ecológicamente relevante para una población, no implica, ipso facto, la identificación de una presión selectiva que pueda considerarse como una causa remota de la evolución en curso de dicha población; tampoco la identificación del rol o la función biológica de un rasgo constituye, por sí misma, ni un análisis de su historia evolutiva, ni la identificación de una presión selectiva que esté incidiendo en la evolución del mismo. Un análisis ecológico, en síntesis, no configura, de por sí, un análisis evolutivo; y la identificación de la relevancia de un rasgo en la ecología, o en el ciclo vital, de un ser vivo, no es lo mismo que explicar dicho rasgo en términos de causas remotas.

Para que esto último pueda ser así, para que se pueda pasar de un nivel de análisis a otro, habría que mostrar, en primer lugar, que existen formas variantes heredables de ese rasgo al interior de la población en estudio; y, en segundo lugar, sería necesario también mostrar cómo, la posesión de una u otra forma de esas formas variantes, dando lugar a modos más o menos eficientes de cumplir con el rol identificado, puede disminuir o incrementar el éxito reproductivo diferencial de sus portadores. Antes de dar ese paso, y esto es algo muy importante de ser destacado, nuestro análisis alude todavía al plano de las causas próximas.

Diferentemente de la presión selectiva que la presencia del arsénico en el ambiente de aquellas plantas puede ejercer sobre la frecuencia y la aptitud relativa de diferentes genotipos al interior de la población, los efectos del arsénico que explican la distribución, la densidad y el crecimiento de los individuos de esa misma población, pueden ser vistos y apuntados sin que sea necesario analizar una secuencia de generaciones. El mapa de la distribución del arsénico, con sus zonas de mayor o menos concentración, y el mapa de la distribución de la población, con sus zonas de mayor o menos densidad y de mayor o menor crecimiento, nos ponen ante un hecho que no es un hecho evolutivo y que podrá ser explicado en virtud de los efectos que esa substancia tiene en el crecimiento de cada planta.

Del mismo modo, el análisis de cómo la posesión de una estructura anatómica, o la realización de un comportamiento, contribuye al cumplimiento del ciclo vital de un organismo en un medio determinado, es algo que debe ser hecho atendiendo a historias individuales de vida y mostrando como las consecuencias de poseer esa 
estructura o cumplir ese comportamiento se reflejan en lo que ocurre con cada organismo individual. Para hacer eso, es cierto, puede ser menester ver y comparar lo que ocurre con varios individuos: si se quiere conocer los hábitos de caza del lobo, puede ser mejor ver lo que pasa con varios lobos, que ver sólo lo que ocurre con uno de ellos. Pero, una vez descubierto un padrón comportamental, y una vez establecido su rol en la vida del lobo, ese comportamiento y sus efectos podrán ser vistos y apuntados, si las condiciones lo permiten, analizando un único caso.

Mientras tanto, si se llegase a descubrir que los lobos de la población estudiada presentan cierta variabilidad hereditaria en un perfil anatómico, y se constata, además, que esa diferencia morfológica conlleva a una realización más o menos eficiente de ese comportamiento, estaríamos detectando una presión selectiva que se traduciría en diferencias de eficacia biológica darwiniana, o de éxito reproductivo diferencial, entre los portadores de las diferentes variantes del rasgo en cuestión; y esa diferencia de eficacia darwiniana, claro; no podría ser verificada analizando o describiendo el ciclo vital, o la historia de vida, de un único espécimen: diferencias o cambios en la eficacia darwiniana son fenómenos que tienen que ver con la evolución de un linaje, y es en ese plano que los mismos deben ser considerados.

O dicho con mayor generalidad: aunque la eficacia darwiniana de un rasgo dependa siempre de aspectos relativos al ciclo vital de los organismos individuales, ella sólo puede ser registrada considerando una secuencia de generaciones ocurrida al interior de un linaje. Es decir: los incrementos o las disminuciones de eficacia biológica son fenómenos estrictamente evolutivos, y no meramente ecológicos, que no pueden apuntarse considerando el ciclo de vida de un organismo individual y que sólo pueden ser explicados por causas remotas. Si por alguna falla en sus mecanismos neurofisiológicos, la curruca de Mayr se quedase a pasar el invierno en New Hampshire, tal vez podríamos observar las marcas que esa situación dejaría en su cuerpo: la perdida de peso, el debilitamiento generalizado, y, tal vez, su agonía y una muerte prematura que lo inhibiría de participar de una nueva temporada de apareamiento. Lo que nunca podríamos registrar, por más que lo pesemos y lo midamos, es la disminución de su eficacia darwiniana.

Sin embargo, el hecho de que exista un hiato y una clara diferencia entre el análisis ecológico, en términos de causas próximas, sobre el rol biológico de un rasgo, y el trazado de su historia evolutiva en términos de causas remotas, no debe hacernos perder de vista que este último no puede existir sin aquél. Las presiones selectivas sólo existen porque hay un fenómeno ecológico llamado lucha por la existencia: los individuos tienen que subsistir y conseguir los medios de subsistencia para crecer y reproducirse en un mundo donde sobran las amenazas y escasean los recursos; y es en ese drama cotidiano, que involucra a cada organismo individual, que debemos remitirnos para comprender las presiones selectivas que pueden estar operado, o pueden haber operado, sobre el linaje que estemos estudiando. Aunque no sea po-

Principia 13(1): 29-50 (2009). 
sible reducir causas remotas a causas próximas, es necesario reconocer que aquéllas no existirian sin éstas (cf. Sterelny \& Griffiths 1999, 254; Cooper 2003, 14; Colyvan 2008, 316).

"La Biología Evolucionaria", como dice Peter Mayhew (2006, 9), "es el campo concernido en entender como los linajes cambian con el transcurso del tiempo (anagénesis), se dividen (cladogénesis), y finalmente se extinguen. "La Ecología", mientras tanto, "está concernida con la interacción de los organismos con su ambiente"; y esa interacción puede ser comprendida en los varios niveles de una jerarquía que va de los individuos a las comunidades, pasando por las propias poblaciones (Mayhew 2006, 9). Pero, sin comprender esa interacción, y por lo menos en lo que respecta a la Teoría de la Selección Natural, ningún proceso evolutivo podría ser explicado. Si el frío y la falta de insectos para comer no afectasen a las currucas individuales, nunca se podría hablar de una presión selectiva que haya propiciado o sostenga ese comportamiento migratorio como respuesta adaptativa a ese problema planteado por el ambiente.

Sin embargo, cuando decimos que ese comportamiento surgió como efecto de una presión selectiva estamos aludiendo a un fenómeno y a una causa que, como ya vimos, sólo pueden ocurrir en el nivel de los linajes. Los organismos no padecen presiones selectivas ni se modifican en respuestas a ellas: ellos pueden sufrir un cambio de temperatura y responder fisiológicamente a esa alteración. Ellos pueden aclimatarse y aprender; pero evolucionar sigue siendo una prerrogativa de los linajes (cf. Sober 1984, 204). Aunque éstos no existen sin los individuos, aun así les ocurren cosas, como sufrir presiones selectivas y evolucionar como respuesta a ellas, que a esos individuos nunca les podrían a ocurrir. Por eso la distinción entre causas próximas y remotas, entendida como una diferencia entre factores que inciden en los estados de los organismos y factores que inciden en la evolución de los linajes, puede y debe ser sostenida no obstante el reconocimiento de que nada podría ocurrir en el plano evolutivo sin que algo ocurra antes en el plano de los vivientes individuales.

\section{El impacto de la ontogenia en la filogenia}

No debe sorprendernos, por eso, que el desarrollo del organismo individual, y no sólo las exigencias ecológicas a las que él está sometido, puedan ser relevantes para el estudio de la evolución. La razón de ello no es difícil de comprender: toda innovación evolutiva posible, toda variación que pueda ofrecerse al escrutinio de la selección natural, tiene que poder corporizarse antes en una alteración ontogenética viable (Amundson 2001, 314; Schwenk \& Wagner 2003, 59; Laubichler 2007, 343). Para que una variación fenotípica surja y pueda entrar en competencia darwiniana con otras, algo en el proceso de la ontogénesis tiene que ser atrofiado o

Principia 13(1): 29-50 (2009). 
hipertrofiado, agregado o suprimido, transpuesto o deformado, postergado o anticipado (cf. Arthur 2004a, 216); y es ahí en donde encuentra su relevancia aquello que Ron Amundson $(2005,176)$ ha llamado Principio de Completud Causal: "Para producir una modificación en la forma adulta, la evolución debe modificar el proceso embriológico responsable por esa forma. Por eso, para comprender la evolución es necesario comprender el desarrollo".

Es que, sea cual sea la índole de esa alteración o de esa reprogramación de la ontogenia, ella tiene que cumplir con dos requisitos fundamentales. En primer lugar ella tiene que ser accesible para el sistema en desarrollo (Maynard Smith et al. 1985, 269; Raff 2000, 78); es decir: tiene que tratarse de una alteración pasible de ser producida en y por ese mismo proceso ontogénetico (Arthur 1997, 48; Azkonobieta $2005,118)$. Y, en segundo lugar, ella tiene que ser tal que, ni aborte ese proceso, ni genere un monstruo totalmente inviable (Amundson 2001a, 320). Además de física o fisiológicamente posible, un cambio evolutivo también tiene que ser ontogenéticamente posible (Amundson 2005, 231; Azkonobieta 2005, 118): la ontogénesis puede o no recapitular a la filogénesis; pero con sus exigencias, que no son menos acuciantes que las ecológicas, la limita y la orienta (cf. Hall 1992, 11; Wilkins 2002, 384).

Lo evolutivamente posible sólo puede ser un recorte de lo ontogenéticamente permitido; y, por eso, la secuencia y el margen de maniobra de los fenómenos evolutivos debe someterse a los constreñimientos y a los direccionamientos que le imponen los requerimientos organizacionales de la ontogenia (Arthur 2004b, 283). Las exigencias de la selección natural pueden hacer muchas cosas con los diferentes linajes de seres vivos sobre las que ella opera; pero, conforme insisten los teóricos de la Biología Evolucionaria del Desarrollo, las marchas y las contramarchas de la selección natural deben ocurrir siempre dentro de los márgenes y siguiendo las secuencias que imponen las exigencias organizacionales del desarrollo orgánico individual. Éste es el locus primero de toda invención morfológica, funcional o comportamental; y, por esa razón, para comprender la evolución su estudio no puede ser menos relevante que el estudio de los factores involucrados en esa lucha por la existencia que es la responsable de la selección natural.

Así, y sobre todo en la medida en que se reconoce que esos constreñimientos y direccionamientos ontogenéticos no sólo limitan sino también ordenan, en parte, la senda de la evolución produciendo un sesgo (Arthur 2004b, 283) en los fenómenos evolutivos que no puede explicarse citando exclusivamente los factores evolutivos clásicos (cf. Moya \& Latorre 2004, 189 y ss), deberemos decir que ellos también constituyen causas remotas o evolutivas. Causas que inciden en la evolución de los linajes y no en el desarrollo de los organismos.

El desarrollo de éstos, es cierto, se ve afectado y usualmente imposibilitado por factores sin los cuales dicho sesgo no existiría. Sin embargo, éste último no puede

Principia 13(1): 29-50 (2009). 
confundirse con aquellos. Un constreñimiento desenvolvimiental, tal como ya fue definido en el consensus paper organizado por Maynard Smith, Dick Burian y Stuart Kauffman en 1985, sería justamente "un sesgo en la producción de variantes fenotípicas o una limitación de la variabilidad fenotípica, causada por la estructura, carácter, composición, o dinámica del sistema desenvolvimiental" (Maynard Smith et al. 1985, 266); y esto ya alude a un fenómeno poblacional y no a un fenómeno organísmico. Lo que constriñe la evolución es un sesgo en la oferta de variantes cuya frecuencia relativa será modificada o preservada por la acción de presiones selectivas o de otros factores como deriva genética o migración. Es decir: la variabilidad a la que alude la teoría de la selección natural es ya un dato poblacional y no organísmico; $y$, por eso, cuando se alude a un sesgo en dicha oferta de variaciones, también se está haciendo referencia a un fenómeno específicamente poblacional.

El desarrollo orgánico se somete a factores y exigencias sin los cuales dicho sesgo no existiría; y la explicación de por qué una determinada reprogramación de la ontogenia resulta inviable para un cierto organismo, corresponde, claro, a ese capítulo de la Biología Funcional que es la Biología del Desarrollo: es en el estudio de la falencia de una ontogenia individual en donde se podrán encontrar y señalar las causas próximas de esa imposibilidad. Pero el sesgo en la oferta de variaciones que condiciona a la evolución no puede confundirse con esos factores y esas exigencias: él es, en todo caso, su reflejo en el nivel poblacional; y en la medida en que ese fenómeno poblacional condicione u oriente la evolución de cierto linaje, diremos que el mismo se ha transformado en un factor evolutivo o en una causa remota que explica por qué dicho linaje evoluciona o evolucionó de la forma en que lo hace o lo hizo.

Del mismo modo en que una presión selectiva no existiría si los organismos individuales no estuviesen sometidos a la lucha por la existencia, los constreñimientos ontogenéticos, considerados como factores evolutivos, no existirían si las ontogenias individuales estuviesen libres de exigencias organizacionales y pudiesen ser alteradas en cualquier dirección y en cualquier secuencia; y, por eso, estudiar esas exigencias, y las secuencias que las mismas imponen para cualquier innovación morfológica, es tan importante para comprender la evolución de un linaje como también lo es, y nunca dejará de serlo, el estudio de los factores ecológicos al que el mismo ha estado sometido (Arthur 2004a, 118).

Pero, del mismo modo en que cuando se estudia el impacto evolutivo de un factor ecológico se hace algo más que mera ecología, cuando se estudia el desarrollo para explicar la ocupación sesgada del morfoespacio generada por un cierto linaje, $o$ la secuencia en que la misma fue realizada, se estará apuntando a un fenómeno que no es individual, que no es meramente ontogenético, que involucra múltiples generaciones consecutivas, y tal vez varias derivaciones de un mismo linaje; y, por eso, se estará apuntando a un fenómeno que no es meramente ontogenético, sino evolutivo o filogenético. La Biología Evolucionaria del Desarrollo, como muy bien lo ha dicho

Principia 13(1): 29-50 (2009). 
Brian Hall $(1992,2)$ estudia "cómo el desarrollo (causalidad próxima) impacta en la evolución (causalidad última) generando cambio evolutivo y cómo el desarrollo mismo ha evolucionado"; pero algo muy semejante podría decirse de la Teoría de la Selección Natural: ella nos lleva a estudiar cómo las relaciones de los organismos con su ambiente (causalidad próxima) impacta en la evolución (causalidad última) generando cambio evolutivo y cómo esa misma relación ha evolucionado como efecto de la selección natural.

\section{La Biología Evolucionaria del Desarrollo como ciencia histórica}

Dije más arriba que, al definir una causa remota como un factor cuya incidencia se registra en la evolución de linajes, estábamos ampliando, y no restringiendo, dicho concepto. Sus contornos quedaban más nítidos, es cierto, pero su cobertura también se ensanchaba porque no sólo las especies constituyen linajes: conforme lo recordé ahí mismo, también son linajes las unidades taxonómicas de orden superior; siempre y cuando, claro, ellas constituyan grupos verdaderamente monofiléticos. Y esto es algo importante de ser tenido en cuenta a la hora de considerar el hecho de que, por lo general, los constreñimientos ontogenéticos presenten la peculiaridad de actuar más allá de los límites de una única especie. Tal es el caso, por ejemplo, de esos constreñimientos que, según suponen los teóricos de la Evo-Devo, son los responsables de que los rasgos homólogos se preserven en un determinado taxón, no obstante las diferentes presiones selectivas a la que las especies que los componen pueden haber estado sometidas a lo largo de millones de años.

En la perspectiva darwiniana, la unidad de tipo se explica en términos de filiación común: ella es algo así como el remanente de uniformidad dejado por una historia de diversificación de las formas cuyo principal motor es la selección natural (Darwin 1859, 206; Amundson 2005, 103). En los diferentes órdenes de un mismo taxón persisten rasgos, o relaciones de correspondencia entre rasgos, homologías (Ghiselin 1997, 205, y 2005, 92), cuya alteración nunca llegó a ser premiada por la selección natural, y por eso se habrían mantenido constantes (Williams 1992, 88). Así, una vez aceptada la Teoría de la Filiación Común, que sirve como telón de fondo de la Teoría de la Selección Natural, la homología acaba recibiendo una explicación que cabría caracterizar como residual: aun siendo una evidencia fundamental para reconstruir el árbol de la vida, ella es lo que sobra una vez que la selección natural ya hizo su trabajo de modificación y diversificación (Amundson 2005, 240).

En la moderna Biología Evolucionaria del Desarrollo, en cambio, la suficiencia de esa concepción meramente vestigial, o histórica, de la unidad de tipo, está siendo cuestionada (Amundson 2005, 239). Para los teóricos de la Evo-Devo, en efecto, las homologías no son el simple remanente de una forma ancestral común (Amundson

Principia 13(1): 29-50 (2009). 
2001b): según ellos insisten, la permanencia de ciertos caracteres, o la constancia de ciertas relaciones entre caracteres, sería explicable como el resultado de constreñimientos ontogenéticos. En ciertos taxones, algunos rasgos o conformaciones particulares tenderían a permanecer, no por el simple hecho de que la selección natural los haya dejado coyunturalmente intocados; sino porque su modificación podría exigir reprogramaciones de la ontogenia muy improbables o incluso imposibles.

Las condiciones de vida, y las presiones selectivas, a las que está sometida cualquier especie de sapos son muy diferentes a las que se somete cualquier especie de murciélago. Pero, no obstante esa diversidad, sus miembros conservan cierta estructura común; y esto, muy posiblemente obedezca al hecho de que cualquier modificación de ese esquema fundamental exigiría una reprogramación ontogenética muy difícil de ser alcanzada. La ontogenia de ambos grupos de tetrápodos parece obedecer a un padrón subyacente común; y es ese padrón el responsable de preservar, en ambos casos, esa estructura y esa disposición general de los miembros que los caracteriza.

Esto, sin embargo, parece plantear una dificultad. Como vimos en el ejemplo anterior, para que los constreñimientos ontogenéticos puedan ser citados como causas positivas de la preservación de los rasgos homólogos, es necesario pensar que ellos actúan sobre diferentes subórdenes de un mismo orden taxonómico aun cuando éstos hayan permanecido reproductivamente aislados durante cientos de millones de años (Amundson 2005, 237); y eso contrasta con lo que ocurre en el caso de los factores evolutivos clásicos como la selección natural, la deriva genética y los procesos migratorios. Éstos son, todos ellos, causas remotas que sólo actúan sobre especies individuales o sobre linajes particulares de éstas; y ahora, al referirnos a los constreñimientos ontogenéticos estamos aludiendo a factores que inciden en el devenir de linajes de un orden superior.

Pero, si se asume que una causa remota es cualquier fenómeno que pueda interferir en el devenir de esas entidades genealógicas, y no meramente ecológicas, que son las poblaciones, en el sentido evolutivo y no ecológico del término, pero también las especies y los grupos taxonómicos de orden superior (cf. Eldredge 1985, 165, y 1995, 179); entonces, podremos reconocer que los constreñimientos ontogenéticos son factores evolutivos en el mismo sentido, y con los mismos derechos, en el que lo son las presiones selectivas originadas por factores ecológicos. Sólo que, a diferencia de estas últimas, la operación de aquéllas, como acabamos de ver, debe registrarse, generalmente, en un plano superior a la especie: auque pueda haber excepciones, en general no parecería necesario apelar a un constreñimiento ontogenético para explicar la posesión de un rasgo por parte de una especie cuyas especies genealógicamente más próximas carecen de él.

No creo, sin embargo, que esto sea una razón para concluir, como Amundson (2005, 229 y ss) sugiere, que los caracteres homólogos sean pensados en Evo-Devo

Principia 13(1): 29-50 (2009). 
como constituyendo clases naturales. En la Biología Evolucionaria del Desarrollo, como en la teoría evolucionaría clásica, la expresión "miembro tetrápodo", al igual que las designaciones de entidades taxonómicas como "león" o "mamífero" (Ghiselin 1983, 105), funciona como el nombre propio de una entidad histórica individual y no como la designación de una clase natural; y, si se afirma que una homología cualquiera es preservada por un mismo mecanismo ontogenético presente en diferentes especies, o incluso en diferentes subphyla como vertebrados y artrópodos (cf. Amundson 2001b), es sólo porque se supone que esas especies o esos subphyla integran, a su vez, un único grupo monofilético que también es considerado como una entidad histórica individual.

Si esta última hipótesis fuese descartada, por más semejante que fuesen esos mecanismos, tal vez se podría hablar de analogías ontogenéticas (cf. Jenner 2006, 390) y las estructuras o rasgos por ellos generados serían considerados como homoplasías, tal como ocurre en el caso de las semejanzas producidas por presiones selectivas análogas. Así, aunque el constreñimiento ontogenético responsable de la preservación de una homología determinada sea una fuerza que, a diferencia de una presión selectiva concreta, actúa más allá de los limites de una especie o de una población individual (cf. Amundson 2005, 235), sus efectos continúan siendo pensados como restrictos a un linaje individual: más amplio que una especie, pero aun así individual (cf. Ghiselin 1997, 306).

Claro, como en cualquier otro capítulo de la Biología Evolucionaria (cf. Sober 2003, 278), en Evo-Devo también existen términos que remiten a clases naturales. Pero los términos que designan rasgos homólogos, diferentemente de aquellos que designan rasgos análogos como las alas de murciélagos y gorriones, no son un buen ejemplo de ello; como tampoco lo son, malgré Quine $(1980,193)$, aquellos términos que, como "León" o "Artrópodos", designan unidades taxonómicas de diferente nivel: ellos son nombres propios que designan ciertas entidades individuales que un día emergieron sobre la faz de la tierra y otro día podrán desaparecer. Es decir: se trata de términos que se parecen más a "Chimborazo" que a "volcán" (cf. Hull 1980, 1984, 1994); y tener esto en cuenta también puede servir para aclarar el malentendido en el que incurre Wallace Arthur (2004a, 160) cuando dice que la Biología Evolucionaria del Desarrollo tendría un perfil más marcadamente teórico, en cuanto que opuesto a histórico, que otras áreas de la Biología Evolucionaria.

Creo, en efecto, que el principal objetivo cognitivo de la Evo-Devo, al igual que el de toda la Biología Evolucionaria, reside, como sabía, en la construcción de narraciones históricas (cf. Hull 1984; López Beltrán 1998) referidas a la evolución y a la constitución de linajes individuales (Mayr 1998c, 91); y no en la construcción de modelos teóricos generales como los que sí pueden encontrarse en la genética de poblaciones. Por eso, y ahora en contra de Ghiselin (2005), tampoco creo que quepa decir que "la Biología Evolucionaria del Desarrollo tiene el potencial de devenir una

Principia 13(1): 29-50 (2009). 
ciencia histórica": ella ya lo es; no obstante la retórica tipológista que, como Jenner (2006) mostró, abunda en los textos de sus cultores y exegetas. Diferentemente de un autor como Brian Goodwin (1998, 191), que pretende darnos "una definición lógica del miembro tetrápodo que es independiente de su historia"; lo que los teóricos de la Biología Evolucionaria del Desarrollo quieren es justamente trazar esa historia (cf. Donoghe 1992, 178); aunque para ellos, claro, esa historia suponga algo más que la simple tensión entre filiación común y selección natural.

\section{Conclusión}

La pregunta de Amundson $(2008,266)$, “inecesitamos una tercera alternativa más allá de próximo-último para referimos a la evolución desenvolvimiental?", puede recibir, en fin, una respuesta negativa: comprender el estatuto de los fenómenos estudiados por la Biología Evolucionaria del Desarrollo, y la naturaleza de la explicación que para ellos se procura, sólo nos exige comprender mejor esa dicotomía; $y$, por los mismos argumentos que aquí se esbozaron para llegar a esa conclusión, también puede decirse que no es posible entender al estudio del desarrollo, en sí mismo, como si fuese un estudio de causas últimas. La Biología del Desarrollo como Mayr siempre insistió, es un capítulo de la Biología Funcional. Un capítulo relevante, como todos los otros, para el estudio de la evolución; pero que también puede y debe ser iluminado por una Biología Evolucionaria que, en ese capítulo suyo denominado Biología Evolucionaria del Desarrollo, analizará, no sólo como los fenómenos ontogenéticos impactan en la evolución, sino también como el propio desarrollo ha evolucionado (cf. Callebaut et al. 2007, 45; Müller, 2007, 506).

Por último, la parte final de la reflexión aquí desarrollada, también nos permite dar una respuesta a la pregunta que Wallace Arthur (2004a, 159) planteó en hacia el final de Biased embryos and evolution: ¿la Evo-Devo debe ser clasificada como Filosofía Natural o como Historia Natural?. Pero, las consideraciones aquí hechas nos llevan a una conclusión contraria a la de Arthur y más favorable a la idea que Mayr (1998c, 92) tenía de la Biología Evolucionaria en general: este nuevo capítulo de los estudios evolucionistas que es la Evo-Devo parece tender más hacia el lado de la Historia Natural que para el lado de la Filosofía Natural. Si el programa hegemónico de la Biología Evolucionaria del Desarrollo siguiese los lineamientos propuestos por Webster y Goodwin (1996), la respuesta sería otra: estaríamos ante el una disciplina mas teórica o nomotética que histórica o ideográfica. Pero en la medida en que esto no sea así, y todo parece indicar que está lejos de serlo, todavía se podrá decir que lo que está surgiendo en los bunkers de la Evo-Devo no es nada más, y nada menos, que un nuevo capítulo de esa historia natural de los seres vivos que, desde 1859, está siendo construida por Biólogos Evolucionarios.

Principia 13(1): 29-50 (2009). 


\section{Bibliografía}

Amundson, R. 2001a. Adaptation and Development: on the lack of a common ground. In Orzack, S. \& Sober, E. (eds.) Adaptationism and Optimality. Cambridge, MA: Cambridge University Press, 303-34.

- 2001b. Homology and Homoplasy: a philosophical perspective. Encyclopedia of Life Sciences. http://www.els.net: Nature Publishing Group.

- 2005. The Changing Role of the Embryo in Evolutionary Thought. Cambridge, MA: Cambridge University Press.

- 2008. Development and Evolution. In Sarkar, S. \& Plutynski, A. (eds) A companion to the Philosophy of Biology. Oxford: Blackwell, 248-68.

Ariew, A. 2003. Ernst Mayr's ultimate/proximate distinction reconsidered and reconstructed. Biology \& Philosophy 18: 553-65.

Arthur, W. 1997. The Origin of Animal Body Plans. Cambridge, MA: Cambridge University Press.

- 2004a. Biased Embryos and Evolution. Cambridge, MA: Cambridge University Press. . 2004b. The effect of development on the direction of evolution: toward a twentyfirst century consensus. Evolution \& Development 6(4): 282-8.

Azkonobieta, T. 2005. Evolución, desarrollo y (auto)organización. Un estudio sobre los principios filosóficos de la Evo-Devo. San Sebastián: Universidad del País Vasco [Tesis Doctoral].

Beatty, J. 1994. The proximate/ultimate distinction in the multiple careers of Ernst Mayr. Biology \& Philosophy 9: 333-56.

Bock, W. \& Wahlert, G. 1998 [1965]. Adaptation and the form-function complex. In Allen, C.; Beckoff, M.; Lauder, G. (eds.) Nature's purposes. Cambridge, MA: MIT Press: 117-67.

Brandon, R. 1990. Adaptation and Environment. Princeton: Princeton University Press.

- 1992. Environment. In Keller, E. \& Lloyd E. (eds.) Keywords in Evolutionary Biology. Cambridge, MA: Harvard University Press, 81-6.

Brewer, R. 1994. The Science of Ecology. New York: Saunders College Publishing.

Callebaut, W.; Müller, G.; Newman, S. 2007. The organismic systems approach: evo-devo and the streamlining of the naturalistic agenda. In Sanson R. \& Brandon R. (eds.) Integrating Evolution and Development. Cambridge, MA: MIT Press, 25-92.

Caponi, G. 2000. Cómo y por qué de lo viviente. Ludus Vitalis 8(14): 67-102.

. 2001. Biología Funcional vs Biología Evolutiva. Episteme 12: 23-46.

- 2003 Experimentos en Biología Evolutiva: ¿qué tienen ellos que los otros no tengan? Episteme 16: 61-97.

- 2007. Física del Organismo vs Hermenéutica del Viviente. História, Ciência, Saúde 14(2): 443-68.

- 2008. Explicación y determinismo en ciencias de la vida. In Tealdi, J. (ed.) Diccionario Latinoamericano de Bioética. Bogotá: UNESCO, 192-5.

Colyvan, M. 2008. Population ecology. In Sarkar, S. \& Plutynski, A. (eds.) A Companion to Philosophy of Biology. Oxford: Blackwell, 301-20.

Cooper, G. 2003. The Science of the Struggle for Existence: on the Foundations of Ecology. Cambridge, MA: Cambridge University Press.

Cronin, H. 1991. The Ant and the Peacock. Cambridge, MA: Cambridge University Press.

Principia 13(1): 29-50 (2009). 
David, P. \& Samadi, S. 2000. La théorie de l'évolution: une logique pour la biologie. Paris: Flammarion.

Donoghue, M. 1992. Homology. In Keller, E. \& Lloyd, E. (eds.) Keywords in Evolutionary Biology. Cambridge, MA: Harvard University Press, 170-9

Eldredge, N. 1985. Unfinished Synthesis: Biological Hierarchies and Modern Evolutionary thought. Oxford: Oxford University Press.

-. 1995. Reinventing Darwin. London: Phoenix.

Gayon, Jean 1992. Darwin et l'après Darwin. Paris: Kimé.

Ghiselin, M. 1983. El triunfo de Darwin. Madrid: Cátedra.

-1997. Metaphysics and the Origin of Species. Cambridge, MA: Harvard University Press.

. 2005. Homology as a relation of correspondence between parts of individuals. Theory in Biosciences 124: 91-103.

Gilbert, S.; Opitz, J.; Raff, R. 1996. Resynthesizing Evolutionary and Developmental Biology. Developmental Biology 173: 357-72.

Goodwin, B. 1998. Las manchas del leopardo. Barcelona: Tusquets.

Gould, S. 2002. The Structure of Evolutionary Theory. Cambridge, MA: Harvard University Press.

Gould, S. \& Vrba, E. 1998 [1982]. Exaptation - a missing term in the science of form. In Allen C.; Beckoff, M.; Lauder, G. (eds.) Nature's Purposes. Cambridge, MA: MIT Press, $519-40$.

Hall, B. 1992. Evolutionary Developmental Biology. London: Chapman \& Hall.

Hull, D. 1980. Individuality and Selection. Annual Review of Ecology and Systematics 11: 311-32.

- 1984. Historical entities and historical narratives. In Hookway, C. (ed.) Minds, Machines and Evolution. Cambridge, MA: Cambridge University Press, 17-42.

- 1994. A matter of individuality. In Sober, E. (ed.) Conceptual Issues in Evolutionary Biology. Cambridge, MA: MIT Press, 193-216.

Jacob, F. 1973. La lógica de lo viviente. Barcelona: Laia.

Jenner, R. 2006. Unburdening Evo-Devo: ancestral attractions, model organisms and basal baloney. Development, Genetics and Evolution 216: 385-94.

Laubichler, M. 2007. Evolutionary Developmental Biology. In Hull, D. \& Ruse, M. (eds.) The Cambridge Companion to the Philosophy of Biology. Cambridge, MA: Cambridge University Press, 342-60.

Lewontin, R. 2000. The Triple Helix. Cambridge, MA: Harvard University Press.

López Beltrán, C. 1998. Narrativa y explicación en las ciencias naturales. In Martinez, S. \& Barahona, A. (eds.) Historia y explicación en biología. México: Fondo de Cultura Económica, 197-211.

Maynard Smith, J.; Burian, R.; Kauffman, S.; Alberch, P.; Campbell, J.; Goodwin, B.; Lande, R.; Raup, D.; Wolpert, L. 1985. Developmental Constraints and Evolution. The Quarterly Review of Biology 60(3): 265-87.

Mayr, E. 1998a [1961]. Causa y efecto en Biología. In Martínez, S. \& Barahona, A. (eds.) Historia y Explicación en Biología. México: Fondo de Cultura Económica, 82-95. 1998b. Así es la Biología. Madrid: Debate.

_ 1998c. O desenvolvimento do pensamento biológico. Brasilia: Editora UnB.

Principia 13(1): 29-50 (2009). 
Moya, A. \& Latorre, A. 2004. Las concepciones internalista y externalista de la evolución biológica. Ludus Vitalis 12(21): 179-96.

Müller, G. 2007. Six memos for Evo-Devo. In Laubichler, M. \& Maienschein, J. (eds.) From Embryology to Evo-Devo. Cambridge, MA: MIT Press, 499-524.

Mayhew, P. 2006. Discovering Evolutionary Ecology. Oxford: Oxford University Press.

Queiroz, K. 1999. The general lineage concept of species and the defining properties of the species category. In Wilson, R. (ed.) Species. Cambridge, MA: MIT Press, 49-90.

Quine, W. 1980. Espécies naturais. In Os Pensadores: Ryle, Strawson, Austin, Quine. São Paulo: Abril, 134-56.

Raff, R. 2000. Evo-devo: the evolution of a new discipline. Nature Reviews Genetics 1: 74-9.

Ruse, M. 1979. La Filosofía de la Biología. Madrid: Alianza.

Schwnenk, K. \& Wagner, G. 2003. Constraint. In Hall, B. \& Olson, W. (eds.) Keywords and Concepts in Evolutionary Developmental Biology. Cambridge, MA: Harvard University Press, 52-60.

Sober, E. 1984. The Nature of Selection. Chicago: The University of Chicago Press.

- 2003. Metaphysical and epistemological issues in modern Darwinian theory. In Hodge, J. \& Radick, G. (eds) The Cambridge Companion to Darwin. Cambridge, MA: Cambridge University Press, 267-89

Stephens, C. 2007. Natural selection. In Mauthen, M. \& Stephens, C. (eds.) Philosophy of Biology. Amsterdam: Elsevier, 111-28.

Sterelny, K. \& Griffiths, P. 1999. Sex and Death. Chicago: The University of Chicago Press.

Tinbergen, N. 1979 [1965]. Conducta y selección natural. In Tinbergen, N. Estudios de Etología II. Madrid: Alianza, 98-118.

- 1985 [1968]. Guerra y paz en los anímales y en el hombre. (Conferencia inaugural de la Cátedra de Comportamiento Animal de la Universidad de Oxford) In Heinz, F. (ed.) Hombre y Animal. Madrid: Orbis, 163-89.

Walsh, D. 2007. Development: three grades of ontogenetic involvement. In Mauthen, M. \& Stephens, C. (eds.) Philosophy of Biology. Amsterdam: Elsevier, 179-200.

Webster, G. \& Goodwin, B. 1996. Form and Transformation: Generative and Relational Principles in Biology. Cambridge, MA.: Cambridge University Press.

Wilkins, A. 2002. The Evolution of Developmental Pathway. Sunderland: Sinauer. 1966. Adaptation and Natural Selection. Princeton: Princeton University Press. 1992. Natural Selection. Oxford: Oxford University Press.

Gustavo Caponi

Rua Esteves Jr. 605, apto.1414 88015-130 Florianópolis SC

Brasil

caponi@cfh.ufsc.br

Resumo. Partindo da premisa de que uma causa próxima é aquela cujos efeitos podem ser registrados nos estados de um organismo individual, sustentarei neste trabalho que o que define uma causa remota é o fato de que seus efeitos possam ser registrados na evolução de linhagens, e não simplesmente em estados populacionais. Isso, por outra parte, não somente nos permitirá entender em que sentido as restrições ontogenéticas devem ser comprendidas

Principia 13(1): 29-50 (2009). 
como causas remotas dos fenômenos evolutivos; mas também nos permitirá colocar em evidência o caráter eminentemente histórico da biologia evolucionaria do desenvolvimento que Wallace Arthur menoscabou em Biased Embryos and Evolution. Assim, e seguindo esta última linha de raciocínio, também procurarei mostrar que, ainda que a ação dessas ditas restrições ontogenéticas possa verificar-se além dos limites de uma população ou espécie particular, isso não implica que estejamos assistindo a um retorno do pensamento tipológico na biologia evolucionária.

Palavras-chave: Biologia evolucionária do desenvolvimento, causas remotas, história natural, pensamento tipológico.

Principia 13(1): 29-50 (2009). 